\title{
GENERIC VANISHING AND MINIMAL COHOMOLOGY CLASSES ON ABELIAN VARIETIES
}

\author{
GIUSEPPE PARESCHI AND MIHNEA POPA
}

\begin{abstract}
We establish a - and conjecture further - relationship between the existence of subvarieties representing minimal cohomology classes on principally polarized abelian varieties, and the generic vanishing of the cohomology of twisted ideal sheaves. The main ingredient is the Generic Vanishing criterion established in [PP3], based on the Fourier-Mukai transform.
\end{abstract}

\section{INTRODUCTION}

This paper is concerned with a relationship between the existence of subvarieties of principally polarized abelian varieties (ppav's) having minimal cohomology class and the (generic) vanishing of certain sheaf cohomology, based on the Generic Vanishing criterion studied in [PP3]. This is in analogy with the well-known equivalence between a subvariety in projective space being of minimal degree and its ideal sheaf being Castelnuovo-Mumford 2-regular (cf. e.g. [EG]). All the statements below are over $\mathbb{C}$. We first state the following conjecture.

Conjecture A. Let $(A, \Theta)$ be a indecomposable ppav of dimension g, and let $X$ be a geometrically nondegenerate closed reduced subscheme of $A$ of pure dimension $d \leq g-2$. The following are equivalent:

(1) X has minimal cohomology class, i.e. $[X]=\frac{\theta^{g-d}}{(g-d) !}$.

(2) $\mathcal{I}_{X}(\Theta)$ is a $G V$-sheaf.

(3) $\mathcal{I}_{X}(2 \Theta)$ satisfies $I T_{0}$.

(4) $\mathcal{O}_{X}(\Theta)$ is $M$-regular, and $\chi\left(\mathcal{O}_{X}(\Theta)\right)=1$.

(5) Either $(A, \Theta)$ is the polarized Jacobian of a smooth projective curve of genus $g$ and $X$ is + or - an Abel-Jacobi embedded copy of $W_{d}(C)$, or $g=5, d=2,(A, \Theta)$ is the intermediate Jacobian of a smooth cubic threefold and $X$ is + or - a translate of the Fano surface of lines.

The condition that $\mathcal{I}_{X}(\Theta)$ be a $G V$-sheaf means that it satisfies a condition analogous to the Green-Lazarsfeld result for canonical bundles GL, namely

$$
\operatorname{codim}\left\{P_{\alpha} \in \operatorname{Pic}^{0}(A) \mid h^{i}\left(\mathcal{I}_{X}(\Theta) \otimes P_{\alpha}\right) \neq 0\right\} \geq i \text { for all } i .
$$

(Cf. $\S 2$ for the notions used in (3) and (4).) The condition of being geometrically nondegenerate is the weakest nondegeneracy condition one usually considers. It is defined in Ra $\S I I$, together with the stronger condition of being nondegenerate (cf. §4) - note however that subvarieties of minimal class are already known to satisfy the stronger version (cf. [Ra], Corollaries II.2 and II.3), so anything giving (1) implies a posteriori that $X$ is in fact nondegenerate.

Key words and phrases. Principally polarized abelian varieties, minimal classes, generic vanishing. 2000 Mathematics Subject Classification: 14K12, 14F17.

MP was partially supported by the NSF grant DMS 0500985 and by an AMS Centennial Fellowship. 
Part (5), more precisely its equivalence with (1), is of course not our conjecture, but rather was formulated in low dimensions by Beauville $\mathrm{Be} 1$ and Ran [Ra], and then in general by Debarre [De1]. In $\S 2$ we explain that most of the implications between the cohomological statements - namely $(2) \Longleftrightarrow(4) \Rightarrow(3)$ - are already known or follow quickly from the definitions. We don't know how to prove that (3) implies the other two. We have already conjectured that (3) is equivalent to (1) before (cf. [PP2], where the analogy with the Castelnuovo-Mumford regularity picture is suggested); we will see here however that condition (2) is more natural in connection with minimal classes. Note also that in [PP1] Proposition 4.4 it is shown that $(5) \Rightarrow$ (4) for $W_{d}$ 's in Jacobians, while in [Hö] the same thing is proved for the Fano surface of a smooth cubic threefold. Thus (5) is known to imply all (1) - (4). Note also that in dimension four we do know the equivalence of (1), (2), (4) and (5), and the fact that they imply (3): indeed, a result of Ran [ Ra asserts the equivalence of (1) and (5) in Conjecture $\mathrm{A}$, while Theorem B below gives that (2) implies (1).

Our main results here are concerned with what is implied by the Generic Vanishing condition. The first is a proof of a slightly stronger $(2) \Rightarrow(1)$ in the Conjecture 1

Theorem B. Let $X$ be a geometrically nondegenerate closed reduced subscheme of pure dimension d of a ppav $(A, \Theta)$ of dimension $g$. If $\mathcal{I}_{X}(\Theta)$ is a $G V$-sheaf, then $X$ is Cohen-Macaulay and $[X]=\frac{\theta^{g-d}}{(g-d) !}$.

The second is a sheaf cohomology criterion for detecting Jacobians. It is the implication $(2) \Rightarrow(5)$ in the Conjecture in the cases $d=1$ and $d=g-2$. The case $d=1$ is in fact an immediate consequence of Theorem B and the Matsusaka-Ran criterion, so the main content is that the same applies for codimension two subvarieties.

Theorem C. Let $X$ be a geometrically nondegenerate equidimensional reduced subscheme of a g-dimensional indecomposable ppav $(A, \Theta)$, of dimension either 1 or $g-2$ respectively. If $\mathcal{I}_{X}(\Theta)$ is a $G V$-sheaf, then $(A, \Theta)$ is the polarized Jacobian of a smooth projective curve $C$ of genus $g$, and $X$ is + or - an Abel-Jacobi embedded copy of either $C$ or $W_{g-2}(C)$ respectively.

There are two new tools that are used for the proofs of Theorems $\mathrm{B}$ and $\mathrm{C}$. The main one is PP3 Theorem $\mathrm{F}$, which equates the $G V$-condition for an object $\mathcal{F}$ in the derived category $\mathbf{D}(A)$ with the vanishing of suitable components of the Fourier-Mukai transform of the Grothendieck dual of $\mathcal{F}$ (cf. $\S 2$ below for a review). The other is to consider systematically the locus of thetatranslates containing $X$, which we call the theta-dual $V(X)$ of $X$ in $\widehat{A}$. As we will show in $\S 5$, such locus supports sheaves which are components of the Fourier-Mukai transform of naturally defined complexes. Roughly speaking, we relate the $G V$-condition for $\mathcal{I}_{X}(\Theta)$ to the cohomology class of $V(X)$ via Grothendieck-Riemann-Roch.

In $\S 8$ we formulate natural geometric conditions on $V(X)$ which should be equivalent to those in Conjecture A. We also note that a proof of $(1) \Rightarrow(2)$ would have a consequence for the implication $(1) \Rightarrow(5)$ as well: one would need to check this only for $X$ of dimension $d \leq\left[\frac{g}{2}\right]$.

Acknowledgements. We are grateful to O. Debarre for pointing out how to arrange things in the proof so that we could assume only geometric nondegeneracy. We also thank Ch. Hacon for suggesting Example 3.2, and to A. Höring for showing us a preliminary version of [Hö]. Finally, we thank the referee for numerous comments which improved the math and the exposition.

\footnotetext{
${ }^{1}$ Note that indecomposability plays no role here, and in fact in anything that is not related to (5).
} 


\section{Preliminaries}

We recall the main Fourier-Mukai terminology and results used in the sequel. If $A$ is an abelian variety of dimension $g$ and $\widehat{A}$ is the dual abelian variety, let $\mathcal{P}$ be a normalized Poincaré line bundle on $A \times \widehat{A}$. The Fourier-Mukai functor [Mu1] is

$$
\mathbf{R} \widehat{\mathcal{S}}: \mathbf{D}(A) \rightarrow \mathbf{D}(\widehat{A}),
$$

the derived functor induced by $\widehat{\mathcal{S}}: \operatorname{Coh}(\mathrm{A}) \rightarrow \operatorname{Coh}(\widehat{A})$, where $\widehat{\mathcal{S}}(\mathcal{F})=p_{\widehat{A} *}\left(p_{A}^{*} \mathcal{F} \otimes \mathcal{P}\right)$. We also consider $\mathbf{R} \mathcal{S}: \mathbf{D}(\widehat{A}) \rightarrow \mathbf{D}(A)$, defined analogously. Mukai's main result (cf. [Mu1], Theorem $2.2)$ is that $\mathbf{R} \widehat{\mathcal{S}}$ is an equivalence of derived categories and

$$
\mathbf{R} \mathcal{S} \circ \mathbf{R} \widehat{\mathcal{S}} \cong\left(-1_{A}\right)^{*}[-g] \text { and } \mathbf{R} \widehat{\mathcal{S}} \circ \mathbf{R} \mathcal{S} \cong\left(-1_{\widehat{A}}\right)^{*}[-g]
$$

An object $\mathcal{F}$ in $\mathbf{D}(A)$ is said to satisfy $W I T_{i}$ (Weak Index Theorem with index $i$ ) if $R^{j} \widehat{\mathcal{S}}(\mathcal{F})=0$, for all $j \neq i$. In this case $R^{i} \hat{\mathcal{S}}(\mathcal{F})$ is denoted by $\widehat{\mathcal{F}}$ and called the Fourier transform of $\mathcal{F}$. Note that $\mathbf{R} \widehat{\mathcal{S}}(\mathcal{F}) \cong \widehat{\mathcal{F}}[-i]$. Moreover, $\mathcal{F}$ satisfies the stronger $I T_{i}$ (Index Theorem with index $i$ ) if

$$
H^{j}\left(A, \mathcal{F} \otimes P_{\alpha}\right)=0 \text { for all } \alpha \in \widehat{A} \text { and all } j \neq i,
$$

where (as in the rest of the paper) we denote by $P_{\alpha} \in \operatorname{Pic}^{0}(A)$ the line bundle corresponding to the point $\alpha \in \widehat{A}$. Reversing the role of $A$ and $\widehat{A}$, and using the functor $\mathbf{R} \mathcal{S}$ instead, all the previous notions/notation can be defined for objects in $\mathbf{D}(\widehat{A})$.

Given an object $\mathcal{F}$ in $\mathbf{D}(A)$ we use the notation

$$
\mathbf{R} \Delta \mathcal{F}:=\mathbf{R} \mathcal{H o m}\left(\mathcal{F}, \mathcal{O}_{A}\right)
$$

and similarly on $\widehat{A}$. Note that the Grothendieck dualizing functor applied to $\mathcal{F}$ is a shift of this, namely $\mathbf{R} \Delta \mathcal{F}[g]$. Grothendieck duality, applied to the present context, states that (cf. [Mu1] $(3.8))$ :

$$
\mathbf{R} \Delta \circ \mathbf{R} \widehat{\mathcal{S}} \cong\left(\left(-1_{\widehat{A}}\right)^{*} \circ \mathbf{R} \widehat{\mathcal{S}} \circ \mathbf{R} \Delta\right)[g], \quad \mathbf{R} \Delta \circ \mathbf{R} \mathcal{S} \cong\left(\left(-1_{A}\right)^{*} \circ \mathbf{R} \mathcal{S} \circ \mathbf{R} \Delta\right)[g]
$$

An object $\mathcal{F}$ in $\mathbf{D}(A)$ is called a $G V$-object (cf. [PP3 $]^{2}$ ) if

$$
\operatorname{codim} \operatorname{Supp}\left(R^{i} \widehat{\mathcal{S}}(\mathcal{F})\right) \geq i \text { for all } i \text {. }
$$

More generally, for any integer $k \geq 0$, an object $\mathcal{F}$ in $\mathbf{D}(A)$ is called a $G V_{k}$-object if

$$
\operatorname{codim} \operatorname{Supp}\left(R^{i} \widehat{\mathcal{S}}(\mathcal{F})\right) \geq i-k \text { for all } i \text {. }
$$

(So $G V=G V_{0}$.). Although not strictly necessary for the purpose of this paper, it is worth recalling that, by [PP3] Lemma 3.8, a coherent sheaf $\mathcal{F}$ is $G V_{k}$ if and only if the more familiar Green-Lazarsfeld condition

$$
\operatorname{codim}_{\widehat{A}} V^{i}(\mathcal{F}) \geq i-k
$$

is satisfied for all $i$, where $V^{i}(\mathcal{F}):=\left\{\alpha \in \widehat{A} \mid H^{i}\left(A, \mathcal{F} \otimes P_{\alpha}\right) \neq 0\right\}$ is the $i$-th cohomological support locus of $\mathcal{F}$. (Same for any object in $\mathbf{D}(A)$ if we use hypercohomology $\mathbf{H}^{i}$.) The main technical tool of the paper is a particular case of the Generic Vanishing criterion [PP3] Theorem F. This holds in a much more general context - for sake of self-containedness, here we state the particular result needed in the paper, for which we provide a short ad hoc proof.

\footnotetext{
${ }^{2}$ In PP3 we define $G V$-objects with respect to a given Fourier-Mukai functor. Since in the present paper we will use only the functor $\mathbf{R} \widehat{\mathcal{S}}$, we suppress this from the notation.
} 
Theorem 2.1. With the notation above, let $\mathcal{F}$ be an object in $\mathbf{D}(A)$, with cohomologies only in non-negative degrees. The following are equivalent:

(a) $\mathcal{F}$ is a $G V_{k}$-object.

(b) $\mathbf{H}^{i}\left(A, \mathcal{F} \otimes \widehat{L^{-1}}\right)=0$ for $i \notin[0, k]$, for any sufficiently positive ample line bundle $L$ on $\widehat{A}$.

(c) $R^{i} \widehat{\mathcal{S}}(\mathbf{R} \Delta \mathcal{F})=0$ for all $i \notin[g-k, g]$.

Proof. (b) $\Leftrightarrow$ (c). To begin with, it is a standard fact that, for a fixed $i$, the vanishing of $R^{i} \widehat{\mathcal{S}}(\mathbf{R} \Delta \mathcal{F})$ is equivalent to

$$
\mathbf{H}^{i}(\widehat{A}, \mathbf{R} \widehat{\mathcal{S}}(\mathbf{R} \Delta \mathcal{F}) \otimes L)=0 \text { for any sufficiently positive ample line bundle } L \text { on } \widehat{A} \widehat{3}
$$

We rewrite

$$
\mathbf{H}^{i}(\widehat{A}, \mathbf{R} \widehat{\mathcal{S}}(\mathbf{R} \Delta \mathcal{F}) \otimes L)=\operatorname{Ext}_{\mathbf{D}(\widehat{A})}^{i}\left(L^{-1}, \mathbf{R} \widehat{\mathcal{S}}(\mathbf{R} \Delta \mathcal{F})\right)=\operatorname{Hom}_{\mathbf{D}(\widehat{A})}\left(L^{-1}, \mathbf{R} \widehat{\mathcal{S}}(\mathbf{R} \Delta \mathcal{F})[i]\right) .
$$

Since $\mathbf{R} \mathcal{S}$ is an equivalence, the last Hom is isomorphic to $\operatorname{Hom}_{\mathbf{D}(A)}\left(\mathbf{R} \mathcal{S}\left(L^{-1}\right), \mathbf{R} \mathcal{S}(\mathbf{R} \widehat{\mathcal{S}}(\mathbf{R} \Delta \mathcal{F}))[i]\right)$. Now $L^{-1}$ is $I T_{g}$ and hence $\mathbf{R} \widehat{\mathcal{S}}\left(L^{-1}\right)=\widehat{L^{-1}}[-g]$. Moreover, by Mukai's theorem (1) we have that $\mathbf{R} \mathcal{S}(\mathbf{R} \widehat{\mathcal{S}}(\mathbf{R} \Delta \mathcal{F})) \cong(-1)_{A}^{*} \mathbf{R} \Delta \mathcal{F}[-g]$. Therefore

$$
\operatorname{Hom}_{\mathbf{D}(A)}\left(\mathbf{R} \mathcal{S}\left(L^{-1}\right), \mathbf{R} \mathcal{S}(\mathbf{R} \widehat{\mathcal{S}}(\mathbf{R} \Delta \mathcal{F}))[i]\right) \cong \operatorname{Hom}_{\mathbf{D}(A)}\left(\widehat{L^{-1}},(-1)_{A}^{*} \mathbf{R} \Delta \mathcal{F}[i]\right) .
$$

The right hand side is isomorphic to $\operatorname{Ext}^{i}\left(\widehat{L^{-1}},(-1)_{A}^{*} \mathbf{R} \Delta \mathcal{F}\right)$, which by Grothendieck-Serre duality is isomorphic to $\mathbf{H}^{g-i}\left(A, \widehat{L^{-1}} \otimes(-1)_{A}^{*} \mathcal{F}\right)$. In conclusion, we have proved that $R^{i} \widehat{\mathcal{S}}(\mathbf{R} \Delta \mathcal{F})$ vanishes if and only if the hypercohomology group $\mathbf{H}^{g-i}\left(A, \widehat{L^{-1}} \otimes(-1)_{A}^{*} \mathcal{F}\right)$ does, for any sufficiently positive ample line bundle $L$ on $\widehat{A}$. This last condition is clearly equivalent to the vanishing of $\mathbf{H}^{g-i}\left(A, \widehat{L^{-1}} \otimes \mathcal{F}\right)$, which proves (b) $\Leftrightarrow(\mathrm{c})$.

(a) $\Rightarrow$ (b). Since $L^{-1}$ is $I T_{g}$, the transform $\widehat{L^{-1}}$ is locally free. The required vanishing for $i<0$ is obvious since $\mathcal{F}$ has cohomologies only in non-negative degrees. Now by Grothendieck duality (2) we have $\left(\widehat{L^{-1}}\right)^{\vee} \cong(-1)_{A}^{*} \widehat{L}$. Therefore

$$
\mathbf{H}^{i}\left(A, \widehat{L^{-1}} \otimes \mathcal{F}\right) \cong \operatorname{Ext}_{\mathbf{D}(A)}^{i}\left((-1)_{A}^{*} \widehat{L}, \mathcal{F}\right) \cong \operatorname{Hom}_{\mathbf{D}(A)}\left((-1)_{A}^{*} \widehat{L}, \mathcal{F}[i]\right) .
$$

Since $\mathbf{R} \widehat{\mathcal{S}}$ is an equivalence, the last Hom is isomorphic to $\operatorname{Hom}_{\mathbf{D}(\widehat{A})}\left(\mathbf{R} \widehat{\mathcal{S}}\left((-1)_{A}^{*} \widehat{L}\right), \mathbf{R} \widehat{\mathcal{S}}(\mathcal{F})[i]\right)$, which is in turn isomorphic to

$$
\operatorname{Hom}_{\mathbf{D}(\widehat{A})}(L[-g], \mathbf{R} \widehat{\mathcal{S}}(\mathcal{F})[i]) \cong \operatorname{Ext}_{D(\widehat{A})}^{g+i}(L, \mathbf{R} \widehat{\mathcal{S}}(\mathcal{F})) \cong \mathbf{H}^{g+i}\left(\widehat{A}, L^{-1} \otimes \mathbf{R} \widehat{\mathcal{S}}(\mathcal{F})\right) .
$$

Here we used Mukai inversion (1) to deduce that $\mathbf{R} \widehat{\mathcal{S}}\left((-1)_{A}^{*} \widehat{L}\right)=\left(\mathbf{R} \widehat{\mathcal{S}} \circ(-1)_{A}^{*} \circ \mathbf{R} \mathcal{S}\right)(L) \cong L[-g]$. Therefore we are reduced to proving that $\mathbf{H}^{g+i}\left(\widehat{A}, L^{-1} \otimes \mathbf{R} \widehat{\mathcal{S}}(\mathcal{F})\right)=0$ for $i>k$ as soon as $\mathcal{F}$ is a $G V_{k}$-object. This follows easily from the hypercohomology spectral sequence

$$
E_{2}^{i j}:=H^{j}\left(\widehat{A}, L^{-1} \otimes R^{i} \widehat{\mathcal{S}}(\mathcal{F})\right) \Rightarrow \mathbf{H}^{i+j}\left(\widehat{A}, L^{-1} \otimes \mathbf{R} \widehat{\mathcal{S}}(\mathcal{F})\right)
$$

since $G V_{k}$ means that the $R^{i} \widehat{\mathcal{S}}(\mathcal{F})$ 's are supported in dimension $\leq g-i+k$, for any $i$.

(c) $\Rightarrow$ (a). Since $\mathbf{R} \Delta$ is an involution on $\mathbf{D}(A)$, Grothendieck duality (2), applied to $\mathbf{R} \Delta \mathcal{F}$, gives

$$
\mathbf{R} \Delta(\mathbf{R} \widehat{\mathcal{S}}(\mathbf{R} \Delta \mathcal{F})) \cong\left(-1_{\widehat{A}}\right)^{*} \mathbf{R} \widehat{\mathcal{S}}(\mathcal{F})[g] .
$$

\footnotetext{
${ }^{3}$ The proof in brief: since $L$ is sufficiently positive, by Serre vanishing the hypercohomology spectral sequence degenerates, hence $\mathbf{H}^{i}(\widehat{A}, \mathbf{R} \widehat{\mathcal{S}}(\mathbf{R} \Delta \mathcal{F}) \otimes L) \cong H^{0}\left(\widehat{A}, R^{i} \widehat{\mathcal{S}}(\mathbf{R} \Delta \mathcal{F}) \otimes L\right)$. But, again by Serre's theorem, the right hand side vanishes if and only if $R^{i} \widehat{\mathcal{S}}(\mathbf{R} \Delta \mathcal{F})$ does.
} 
This gives rise to a spectral sequence

$$
E_{2}^{i j}:=\mathcal{E} x t^{i+j}\left(R^{j} \widehat{\mathcal{S}}(\mathbf{R} \Delta \mathcal{F}[g]), \mathcal{O}_{\widehat{A}}\right) \Rightarrow(-1)_{\widehat{A}}^{*} R^{i} \widehat{\mathcal{S}}(\mathcal{F})
$$

The hypothesis is that $R^{j} \widehat{\mathcal{S}}(\mathbf{R} \Delta \mathcal{F})$ vanish for $j \notin[g-k, g]$, i.e. that the $R^{j}:=R^{j} \widehat{\mathcal{S}}(\mathbf{R} \Delta \mathcal{F}[g])$ vanish for $j \notin[-k, 0]$. One knows that

$$
\operatorname{codim} \operatorname{Supp}\left(\mathcal{E} x t^{i+j}\left(R^{j}, \mathcal{O}_{Y}\right)\right) \geq i+j
$$

for all $i$ and $j$. This is a particular case of the following general fact: for any $h$, the $\mathcal{E} x t^{h}\left(\mathcal{G}, \mathcal{O}_{X}\right)$ sheaf associated to a sheaf $\mathcal{G}$ on a smooth variety $X$ is always supported in codimension $\geq h$ (see e.g. OSS, Ch.II, Lemma 1.1.2). Since the only non-zero $R^{j}$-sheaves are for $j \geq-k$, we have that the codimension of the support of every $E_{\infty}$ term of the above spectral sequence is at least $i-k$. This implies immediately that $R^{i} \widehat{\mathcal{S}}(\mathcal{F})=0$ for $i<0$ and $\operatorname{codim} \operatorname{Supp}\left(R^{i} \widehat{\mathcal{S}}(\mathcal{F})\right) \geq i-k$, for all $i \geq 0$.

An important particular case is that of $k=0$, when the result says that $\mathcal{F}$ is $G V$ if and only if $\mathbf{R} \Delta \mathcal{F}$ satisfies $W I T_{g}$. In this case, the fact that (c) is equivalent to (b) and implies (a) appears already in $\mathrm{Hac}$.

Finally, we recall that a sheaf on $A$ is called $M$-regular (cf. [PP1]) if

$$
\operatorname{codim} \operatorname{Supp}\left(R^{i} \widehat{\mathcal{S}}(\mathcal{F})\right)>i \text { for all } i>0 \text {. }
$$

\section{IMPLiCATIONS BETWEEN THE COHOMOLOGICAL STATEMENTS}

The implication $(2) \Rightarrow(3)$ in Conjecture $\mathrm{A}$ follows from the general Lemma below, which is proved exactly as the implication $(1) \Rightarrow(2)$ of Theorem 2.1 (note that $\left.\widehat{\mathcal{O}_{\widehat{A}}(-\Theta}\right)=\left(-1_{A}\right)^{*} \mathcal{O}_{A}(\Theta)$ - cf. [Mu1, Proposition 3.11). In $\S 7$ below we will see some extra implications of the $G V$ hypothesis when $\mathcal{F}$ is (the twist of) an ideal sheaf.

Lemma 3.1. Let $\mathcal{F}$ be a $G V$-sheaf on a ppav $(A, \Theta)$. Then $\mathcal{F}(\Theta)$ satisfies $I T_{0}$.

Example 3.2. Given Lemma 3.1, it is natural to ask whether the implication $(3) \Rightarrow(2)$ might also hold for an arbitrary coherent sheaf $\mathcal{F}$ instead of $\mathcal{I}_{X}(\Theta)$. We give an example showing that this is not the case, so this implication (if true) should be more geometric. We thank Christopher Hacon for suggesting this example. Consider $(A, \Theta)$ any ppav, say with $\Theta$ symmetric for simplicity, $k \geq 2$ an integer, and $\phi_{k}: A \rightarrow A$ the map given by multiplication by $k$. Then $\mathcal{F}:=\phi_{k *} \mathcal{O}_{A}(-\Theta)$ is a sheaf which is not $G V$, but $\mathcal{F}(\Theta)$ satisfies $I T_{0}$. Indeed $\mathcal{F}(\Theta)$ satisfies $I T_{0}$ if and only if $\mathcal{O}_{A}(-\Theta) \otimes \phi_{k}^{*} \mathcal{O}_{A}(\Theta)$ does so, which is obviously true since $\phi_{k}^{*} \mathcal{O}_{A}(\Theta) \cong \bigoplus \mathcal{O}_{A}\left(k^{2} \Theta\right)$. On the other hand, $\mathcal{F}$ is not $G V$ since

$$
H^{g}\left(A, \mathcal{F} \otimes P_{\alpha}\right) \cong H^{g}\left(A, \mathcal{O}_{A}(-\Theta) \otimes \phi_{k}^{*} P_{\alpha}\right)
$$

which is non-zero for all $\alpha$.

The equivalence $(2) \Longleftrightarrow(4)$ follows directly from the definitions, without any assumptions on $X$.

Lemma 3.3. Let $(A, \Theta)$ be a ppav and $X$ a closed subscheme of $A$. The following are equivalent:

(a) $\mathcal{I}_{X}(\Theta)$ is a $G V$-sheaf.

(b) $\mathcal{O}_{X}(\Theta)$ is $M$-regular and $\chi\left(\mathcal{O}_{X}(\Theta)\right)=1$. 
Proof. Applying the Fourier-Mukai functor to the exact sequence

$$
0 \longrightarrow \mathcal{I}_{X}(\Theta) \longrightarrow \mathcal{O}_{A}(\Theta) \longrightarrow \mathcal{O}_{X}(\Theta) \longrightarrow 0,
$$

we obtain that $R^{i} \widehat{\mathcal{S}}\left(\mathcal{O}_{X}(\Theta)\right) \cong R^{i+1} \widehat{\mathcal{S}}\left(\mathcal{I}_{X}(\Theta)\right)$ for all $i \geq 1$, and

$$
0 \longrightarrow \mathcal{O}_{A}(-\Theta) \longrightarrow R^{0} \widehat{\mathcal{S}}\left(\mathcal{O}_{X}(\Theta)\right) \longrightarrow R^{1} \widehat{\mathcal{S}}\left(\mathcal{I}_{X}(\Theta)\right) \longrightarrow 0
$$

since $R^{0} \widehat{\mathcal{S}}\left(\mathcal{I}_{X}(\Theta)\right)$ being torsion must therefore be zero. Thus if $\mathcal{I}_{X}(\Theta)$ is a $G V$-sheaf, then $\mathcal{O}_{X}(\Theta)$ is $M$-regular and $R^{0} \widehat{\mathcal{S}}\left(\mathcal{O}_{X}(\Theta)\right)$ has generic rank 1 , which by base change is equivalent to $h^{0}\left(A, \mathcal{O}_{X}(\Theta) \otimes P_{\alpha}\right)=1$ for $P_{\alpha} \in \operatorname{Pic}^{0}(A)$ general, i.e. $\chi\left(\mathcal{O}_{X}(\Theta)\right)=1$. Conversely, if (b) holds than the same reasoning shows that we need to worry only about $R^{1} \widehat{\mathcal{S}}\left(\mathcal{I}_{X}(\Theta)\right)$. But this must be supported on a proper subset, since the argument above can be completely reversed.

\section{The theta-DUAL of A SUbVariety of A PPAV}

Let $(A, \Theta)$ be a ppav of dimension $g$. Consider a closed reduced subscheme $X$ of $A$, of dimension $d \leq g-2$. We put a natural scheme structure on the locus of theta-translates containing $X$.

Lemma 4.1. For any $\alpha \in \widehat{A}$ there is a canonical identification

$$
\left(-1_{\widehat{A}}\right)^{*} R^{g} \widehat{\mathcal{S}}\left(\mathbf{R} \Delta\left(\mathcal{I}_{X}(\Theta)\right)\right) \otimes_{\mathcal{O}_{\widehat{A}, \alpha}} \mathbb{C}(\alpha) \cong H^{0}\left(A, \mathcal{I}_{X}(\Theta) \otimes P_{\alpha}\right)^{\vee} .
$$

Proof. We apply base change for complexes, as in [EGA III] 7.7: since $\mathbf{H}^{g+1}\left(A, \mathbf{R} \Delta\left(\mathcal{I}_{X}(\Theta)\right) \otimes\right.$ $\left.P_{\alpha}\right)=0$ for all $P_{\alpha} \in \operatorname{Pic}^{0}(A)$, we have for any $\alpha$ a natural isomorphism

$$
\left(-1_{\widehat{A}}\right)^{*} R^{g} \widehat{\mathcal{S}}\left(\mathbf{R} \Delta\left(\mathcal{I}_{X}(\Theta)\right)\right) \otimes_{\mathcal{O}_{\widehat{A}, \alpha}} \mathbb{C}(\alpha) \cong \mathbf{H}^{g}\left(A, \mathbf{R} \Delta\left(\mathcal{I}_{X}(\Theta)\right) \otimes P_{\alpha}^{-1}\right) .
$$

By Grothendieck-Serre duality, this hypercohomology group is isomorphic to $H^{0}\left(A, \mathcal{I}_{X}(\Theta) \otimes\right.$ $\left.P_{\alpha}\right)^{\vee}$.

Definition 4.2. We denote by $V(X)$ the scheme-theoretic support of $\left(-1_{\widehat{A}}\right)^{*} R^{g} \widehat{\mathcal{S}}\left(\mathbf{R} \Delta\left(\mathcal{I}_{X}(\Theta)\right)\right)$, and call it the theta-dual of $X$ (with respect to the principal polarization $\Theta$ ). By Lemma 4.1, set-theoretically we have

$$
V(X)=\left\{\alpha \mid h^{0}\left(A, \mathcal{I}_{X}(\Theta) \otimes P_{\alpha}\right) \neq 0\right\} \subset \widehat{A},
$$

which via the principal polarization is identified with the locus $\left\{a \in A \mid X \subset \Theta_{a}\right\}$ of thetatranslates containing $X$. More precisely we have

Corollary 4.3. The sheaf $\left(-1_{\widehat{A}}\right)^{*} R^{g} \widehat{\mathcal{S}}\left(\mathbf{R} \Delta\left(\mathcal{I}_{X}(\Theta)\right)\right)$ is a line bundle on $V(X)$. In fact

$$
\left(-1_{\widehat{A}}\right)^{*} R^{g} \widehat{\mathcal{S}}\left(\mathbf{R} \Delta\left(\mathcal{I}_{X}(\Theta)\right)\right) \cong \mathcal{O}_{V(X)}(\Theta)
$$

Proof. The first assertion follows from the previous Lemma: the fibers of $\left(-1_{\widehat{A}}\right)^{*} R^{g} \widehat{\mathcal{S}}\left(\mathbf{R} \Delta\left(\mathcal{I}_{X}(\Theta)\right)\right)$ have dimension either zero or one, and the latter happens if and only if $\alpha \in V(X)$. For the last assertion, note that we have a natural surjective homomorphism

$$
R^{g} \widehat{\mathcal{S}}\left(\mathbf{R} \Delta\left(\mathcal{O}_{A}(\Theta)\right)\right) \longrightarrow R^{g} \widehat{\mathcal{S}}\left(\mathbf{R} \Delta\left(\mathcal{I}_{X}(\Theta)\right)\right) .
$$

Indeed, as in Lemma 4.1, the fiber over $\alpha$ is given by the dual of the injection $H^{0}\left(A, \mathcal{I}_{X}(\Theta) \otimes\right.$ $\left.P_{\alpha}\right) \hookrightarrow H^{0}\left(A, \mathcal{O}_{A}(\Theta) \otimes P_{\alpha}\right)$. Now $\mathbf{R} \Delta\left(\mathcal{O}_{A}(\Theta)\right) \cong \mathcal{O}_{A}(-\Theta)$ and $\left(-1_{\widehat{A}}\right)^{*} R^{g} \widehat{\mathcal{S}}\left(\mathcal{O}_{A}(-\Theta)\right) \cong \mathcal{O}_{\widehat{A}}(\Theta)$, which gives the conclusion. 
Recall from [ $\mathrm{Ra}$ ] $\S \mathrm{II}$ that an equidimensional reduced subscheme $X$ is nondegenerate in $A$ if the kernel of the restriction map $H^{0}\left(A, \Omega_{A}^{d}\right) \rightarrow H^{0}\left(X_{\text {reg }}, \Omega_{X_{\text {reg }}}^{d}\right)$ is 0 , and geometrically nondegenerate if the same kernel contains no nonzero decomposable $d$-forms. It is enough here to work with geometrically nondegenerate subschemes 4 Given that the image of the difference map $\phi: X \times V(X) \rightarrow A,(x, y) \rightarrow x-y$, is contained in $\Theta$, the following is a special case of De2] Proposition 1.4.

Lemma 4.4. Let $X$ be a geometrically nondegenerate equidimensional reduced subscheme of $A$, of dimension $d$. Then

$$
\operatorname{dim} V(X) \leq g-d-1
$$

Example 4.5. If $C$ is a smooth projective curve of genus $g$, consider the image $W_{d}$ of the $d$-th symmetric product of $C$ via some Abel-Jacobi map. Then it is folklore, and straightforward to verify, that set-theoretically $V\left(W_{d}\right)=-W_{g-d-1}$ up to translate. We will see later that this actually holds scheme-theoretically, and that a similar fact holds for the Fano surface (cf. §8.1).

\section{Consequences of the Generic Vanishing CRIterion}

We consider now $X$ to be a closed reduced subscheme of $A$ of pure dimension $d$. We draw a number of sheaf-theoretic consequences in the case when $\mathcal{I}_{X}(\Theta)$ is a $G V$-sheaf, based on the criterion in $\S 2$. Recall that by Theorem 2.1 this is equivalent to the fact that the complex $\mathbf{R} \Delta\left(\mathcal{I}_{X}(\Theta)\right)$ satisfies $W I T_{g}$ which, together with Corollary 4.3, means that

$$
\mathbf{R} \widehat{\mathcal{S}}\left(\mathbf{R} \Delta\left(\mathcal{I}_{X}(\Theta)\right)\right)=R^{g} \widehat{\mathcal{S}}\left(\mathbf{R} \Delta\left(\mathcal{I}_{X}(\Theta)\right)\right)[-g] \cong\left(-1_{\widehat{A}}\right)^{*} \mathcal{O}_{V(X)}(\Theta)[-g] .
$$

Proposition 5.1. Assume that $\mathcal{I}_{X}(\Theta)$ is a $G V$-sheaf. Then:

(a) $\operatorname{dim} V(X) \geq g-d-1$.

(b) If equality is attained, i.e. $\operatorname{dim} V(X)=g-d-1$, then $V(X)$ is Cohen-Macaulay and equidimensional if and only if $\mathcal{I}_{X}(\Theta)$ satisfies $W I T_{d+1}$.

Proof. (a) Note first that

$$
\operatorname{codim}_{\widehat{A}} V(X)=\min \left\{k \mid \mathcal{E} x t_{\mathcal{O}_{\widehat{A}}}^{k}\left(\mathcal{O}_{V(X)}, \mathcal{O}_{\widehat{A}}\right) \neq 0\right\} .
$$

On the other hand, we claim that $\mathbf{R} \widehat{\mathcal{S}}\left(\mathcal{I}_{X}(\Theta)\right) \cong \mathbf{R} \Delta\left(\mathcal{O}_{V(X)}(\Theta)\right)$, which implies for all $i$ that

$$
\mathcal{E} x t_{\mathcal{O}_{\widehat{A}}}^{i}\left(\mathcal{O}_{V(X)}(\Theta), \mathcal{O}_{\widehat{A}}\right) \cong R^{i} \widehat{\mathcal{S}}\left(\mathcal{I}_{X}(\Theta)\right)
$$

To prove the claim, start with (4) above and apply the functor $\left(-1_{\widehat{A}}\right)^{*} \circ \mathbf{R} \Delta \circ[g]$ to both sides. On the right hand side we obtain $\mathbf{R} \Delta\left(\mathcal{O}_{V(X)}(\Theta)\right)$. On the left hand side we have

$$
\mathbf{R} \Delta\left(\left(-1_{\widehat{A}}\right)^{*} \mathbf{R} \widehat{\mathcal{S}} \mathbf{R} \Delta\left(\mathcal{I}_{X}(\Theta)[g]\right)\right) \cong \mathbf{R} \Delta\left(\mathbf{R} \Delta \mathbf{R} \widehat{\mathcal{S}}\left(\mathcal{I}_{X}(\Theta)\right)\right) \cong \mathbf{R} \widehat{\mathcal{S}}\left(\mathcal{I}_{X}(\Theta)\right),
$$

where for the first isomorphism we used Grothendieck duality (2).

Now since $X$ has dimension $d$, we have that $R^{i} \widehat{\mathcal{S}}\left(\mathcal{O}_{X}(\Theta)\right)=0$ for $i>d$, and so $R^{i} \widehat{\mathcal{S}}\left(\mathcal{I}_{X}(\Theta)\right)=$ 0 for $i>d+1$, by applying the Fourier-Mukai functor to the exact sequence

$$
0 \rightarrow \mathcal{I}_{X}(\Theta) \rightarrow \mathcal{O}_{A}(\Theta) \rightarrow \mathcal{O}_{X}(\Theta) \rightarrow 0 .
$$

By the above this implies that the dimension of $V(X)$ is at least $g-d-1$.

\footnotetext{
${ }^{4}$ We thank O. Debarre for pointing this out.
} 
(b) This follows directly from (5), as it is well known that the condition on $V(X)$ is equivalent to the fact that

$$
\mathcal{E}_{x} t_{\mathcal{O}_{\widehat{A}}}^{k}\left(\mathcal{O}_{V(X)}, \mathcal{O}_{\widehat{A}}\right) \neq 0 \Longleftrightarrow k=d+1
$$

Theorem 5.2. Assume that $\mathcal{I}_{X}(\Theta)$ is a $G V$-sheaf and $X$ is geometrically nondegenerate. Then:

(a) $V(X)$ is Cohen-Macaulay of pure dimension $g-d-1$.

(b) $\mathcal{I}_{V(X)}(\Theta)$ is a $G V$-sheaf.

(c) $V(V(X))=X$.

(d) $X$ is Cohen-Macaulay.

Proof. (a) Note first that $\operatorname{dim} V(X)=g-d-1$, since by Proposition 5.1 (a) it is at least $g-d-1$, while by Lemma 4.4 it is at most that much. But then by Proposition [5.1(b), the conclusion holds if we show that $\mathcal{I}_{X}(\Theta)$ satisfies $W I T_{d+1}$.

This is the key point in the proof of the entire theorem: we apply Theorem 2.1 to the complex $\mathcal{F}=\mathbf{R} \Delta\left(\mathcal{I}_{X}(\Theta)\right)$ (so that $\mathbf{R} \Delta \mathcal{F}=\mathcal{I}_{X}(\Theta)$ ). We know that $\mathcal{F}$ is $G V_{g-d-1}$ in a very strong sense: $R^{i} \widehat{\mathcal{S}} \mathcal{F}=0$ for $i \neq g$, while $\operatorname{codim}_{\widehat{A}} \operatorname{Supp}\left(R^{g} \widehat{\mathcal{S}} \mathcal{F}\right)=\operatorname{codim}_{\widehat{A}} V(X)=d+1=g-(g-d-1)$. Thus in Theorem 2.1 we can take $k=d-g-1$. The implication (a) $\Rightarrow$ (c) in the theorem gives that $R^{i} \widehat{\mathcal{S}}\left(\mathcal{I}_{X}(\Theta)\right)=R^{i} \widehat{\mathcal{S}}(\mathbf{R} \Delta \mathcal{F})=0$ for $i<d+1$. On the other hand we noted above that we have $R^{i} \widehat{\mathcal{S}}\left(\mathcal{I}_{X}(\Theta)\right)=0$ for $i>d+1$, simply since $X$ is $d$-dimensional.

(b) Again by Theorem 2.1, this is the same as showing that $\mathbf{R} \Delta\left(\mathcal{I}_{V(X)}(\Theta)\right)$ satisfies $W I T_{g}$. We prove more precisely that

$$
\mathbf{R} \mathcal{S}\left(\mathbf{R} \Delta\left(\mathcal{I}_{V(X)}(\Theta)\right)\right) \cong\left(-1_{A}\right)^{*} \mathcal{O}_{X}(\Theta)
$$

Using Mukai's inversion theorem for the Fourier functor, i.e. equation (1) in $\S 2$, this statement is equivalent to showing that

$$
\mathbf{R} \widehat{\mathcal{S}}\left(\mathcal{O}_{X}(\Theta)\right) \cong \mathbf{R} \Delta\left(\mathcal{I}_{V(X)}(\Theta)\right) .
$$

Because of the short exact sequence $0 \longrightarrow \mathcal{I}_{X}(\Theta) \longrightarrow \mathcal{O}_{A}(\Theta) \longrightarrow \mathcal{O}_{X}(\Theta) \longrightarrow 0$ and its analogue for $V(X)$, this is in turn equivalent to

$$
\mathbf{R} \widehat{\mathcal{S}}\left(\mathcal{I}_{X}(\Theta)\right) \cong \mathbf{R} \Delta\left(\mathcal{O}_{V(X)}(\Theta)\right) .
$$

This was shown in the proof of Proposition 5.1 .

(c) The assertion follows immediately from (6) above, since as in Lemma 4.1 the Fourier transform of $\mathbf{R} \Delta\left(\mathcal{I}_{V(X)}(\Theta)\right)$ is supported on $-V(V(X))$.

(d) From (a) we know that $V(X)$ is equidimensional, and from (b) that $\mathcal{I}_{V(X)}(\Theta)$ is a $G V$-sheaf. Since we know already that $\operatorname{dim} V(X)=g-d-1$ and $\operatorname{dim} V(V(X))=\operatorname{dim} X=d$, we can deduce as in (a) (without assuming nondegeneracy for $V(X))$ that $\mathcal{I}_{V(X)}(\Theta)$ satisfies $W I T_{g-d}$. Proposition 5.1(b) implies then that $X$ is Cohen-Macaulay.

Remark 5.3. Since $X$ is Cohen-Macaulay, it is a posteriori not hard to understand the main technical tool, namely the object $\mathbf{R} \Delta\left(\mathcal{I}_{X}(\Theta)\right)$, better: in fact it is represented by a complex with two non-zero cohomology sheaves: $\mathcal{O}_{A}(-\Theta)$ in degree 0 and $\omega_{X}(-\Theta)$ in degree $g-d-1$. 
Corollary 5.4. If $X$ is geometrically nondegenerate and $\mathcal{I}_{X}(\Theta)$ is a $G V$-sheaf, then $\omega_{X}(-\Theta)$ satisfies $W I T_{d}$ and

$$
\widehat{\omega_{X}(-\Theta)} \cong\left(-1_{\widehat{A}}\right)^{*} \mathcal{I}_{V(X)}(\Theta)
$$

Also $\omega_{V(X)}(-\Theta)$ satisfies $W I T_{g-d-1}$ and

$$
\widehat{\omega_{V(X)}(-\Theta)} \cong\left(-1_{A}\right)^{*} \mathcal{I}_{X}(\Theta)
$$

Proof. First of all, since we've seen that $X$ and $V(X)$ are Cohen-Macaulay, they do have dualizing sheaves. The second assertion is the same as the first, with the roles of $X$ and $V(X)$ reversed. For the first, note that $\mathbf{R} \Delta\left(\omega_{X}(-\Theta)\right) \cong \mathcal{O}_{X}(\Theta)[g-d]$. Hence the statement follows from the isomorphism (7) above and Grothendieck duality (2), as in the proof of Proposition 5.1 .

Remark 5.5. The results of this section imply that if the $G V$ condition is satisfied for $\mathcal{I}_{X}(\Theta)$ then the role of the schemes $X$ and $V(X)$ is symmetric: any type of result we are interested in which is true for $X$ holds also for $V(X)$, and conversely. Note that Theorem 6.1 below implies that $V(X)$ is in fact nondegenerate, and so the same thing holds for $X$.

\section{Minimal Cohomology Classes}

In this section we prove a result which, by the symmetry noted in Remark 5.5, is equivalent to Theorem B. Besides the results of the previous section, we will use applications of the Grothendieck-Riemann-Roch theorem.

Theorem 6.1. Let $X$ be a geometrically nondegenerate closed reduced subscheme of pure dimension d of a ppav $(A, \Theta)$ of dimension $g$, such that $\mathcal{I}_{X}(\Theta)$ is a $G V$-sheaf. Then $[V(X)]=\frac{\theta^{d+1}}{(d+1) !}$.

Proof. Recall from Corollary 5.4 that $\left(-1_{A}\right)^{*} \omega_{X}(-\Theta)$ satisfies $W I T_{d}$, and its Fourier-Mukai transform is $\mathcal{I}_{V(X)}(\Theta)$, which satisfies $W I T_{g-d}$. We will use the relationship in cohomology between the Chern character of a WIT-sheaf and that of its Fourier-Mukai transform, established by Mukai [Mu2] Corollary 1.18 as an application of Grothendieck-Riemann-Roch. In our situation, for the $(d+1)$-st component of the Chern character (indexed by the codimension), this is written as

$$
\operatorname{ch}_{d+1}\left(\mathcal{I}_{V(X)}(\Theta)\right)=(-1)^{g+1} P D\left(\operatorname{ch}_{g-d-1}\left(\left(-1_{A}\right)^{*} \omega_{X}(-\Theta)\right)\right)
$$

where $P D$ denotes the Poincaré duality isomorphism, and we ignore notation for the inclusion map of $X$ into $A$.

We now compute the two sides. On one hand, since $\omega_{X}(-\Theta)$ is supported on $X$, which has dimension $d$, it is standard that $\operatorname{ch}_{g-d-1}\left(\left(-1_{A}\right)^{*} \omega_{X}(-\Theta)\right)=0$ since the Chern character $\operatorname{ch}\left(\omega_{X}(-\Theta)\right)$ has no non-zero components in degree less than the codimension $g-d$. (For example this is a simple consequence of Grothendieck-Riemann-Roch - cf. e.g. [Fu] Example 18.3.11.)

On the other hand

$$
\begin{gathered}
\operatorname{ch}\left(\mathcal{I}_{V(X)}(\Theta)\right)=\operatorname{ch}\left(\mathcal{O}_{\widehat{A}}(\Theta)\right)-\operatorname{ch}\left(\mathcal{O}_{V(X)}(\Theta)\right)= \\
=\left(1+\theta+\frac{\theta^{2}}{2 !}+\ldots\right)-([V(X)]+\ldots),
\end{gathered}
$$

\footnotetext{
${ }^{5}$ This says that if $\mathcal{F}$ is a sheaf on $A$ satisfying $W I T_{j}$, then $\operatorname{ch}_{i}(\widehat{\mathcal{F}})=(-1)^{i+j} P D_{2 g-2 i}\left(\operatorname{ch}_{g-i}(\mathcal{F})\right)$.
} 
The second term starts in $C H^{d+1}$; we apply the same reasoning as in the previous paragraph, in the more precise form saying that $\operatorname{ch}\left(\mathcal{O}_{V(X)}\right)$ starts with the class $[V(X)]$ (cf. [Fu] Example 15.2.16), using the fact that by Theorem $5.2(\mathrm{a}), V(X)$ is of pure dimension $g-d-1$. We obtain

that $\operatorname{ch}_{d+1}\left(\mathcal{I}_{V(X)}(\Theta)\right)=\frac{\theta^{d+1}}{(d+1) !}-[V(X)]$. This has to be 0 by the paragraph above, which gives the conclusion.

\section{Characterization of Jacobians via Generic Vanishing For extremal Dimensions}

In this section we prove Theorem $\mathrm{C}$. At this stage it is in fact an immediate corollary of Theorem 6.1 and the Matsusaka-Hoyt criterion [Ho], Theorem 1.

Proof. (of Theorem C.) By Theorem6.1 and Theorem 5.2 we know that both $X$ and $V(X)$ have minimal class. If $X$ is a curve, then the statement follows directly from the Matsusaka criterion.

If $X$ has codimension 2, then $V(X)$ is a 1-cycle of minimal class. By the MatsusakaHoyt criterion we obtain that $V(X)$ is an irreducible Abel-Jacobi embedded curve $C$, since otherwise $(A, \Theta)$ would have to split into a product of polarized Jacobians, which contradicts indecomposability. Now given any Abel-Jacobi embedded curve $C$, by Example 4.5 we have $V(C)=-W_{g-2}(C)$ in the dual Jacobian. Since $V(V(X))=X$ by Theorem 5.2 (c), this concludes the proof. (The entire discussion is of course up to + or - a translate).

Remark 7.1. The exact same proof tells us what happens in case $(A, \Theta)$ is decomposable as well. The Matsusaka-Hoyt theorem implies that $A$ is a product of Jacobians and either $X$ or $V(X)$, according to which has dimension 1, is a reduced 1-cycle which projects to an Abel-Jacobi embedded curve on each one of the factors.

\section{Complements}

8.1. The theta-dual of $W_{d}$ and of the Fano surface. Going back to Example 4.5, we can now say that $V\left(W_{d}\right)=-W_{g-d-1}$ scheme-theoretically. The equality is known set-theoretically, and by Theorem $5.2(\mathrm{a}), V\left(W_{d}\right)$ has no embedded components. Since $\left[W_{g-d-1}\right]=\frac{\theta^{d+1}}{(d+1) !}$, the conclusion follows from Theorem B.

It was shown in [CG that for the Fano surface of lines $F$ in the intermediate Jacobian of a smooth cubic threefold we have $F-F=\Theta$ set-theoretically, and $[F]=\frac{\theta^{3}}{3 !}$. The first equality gives $F \subset V(F)$, so given the results above $V(F)$ has dimension 2 and $F$ as a component. A priori it could have other components too. The results of [Hö] imply however that $\mathcal{I}_{F}(\Theta)$ is $G V$, which again by Theorem 5.2 (a) and Theorem $\mathrm{B}$ gives that $V(F)$ is equidimensional and $[V(F)]=\frac{\theta^{3}}{3 !}$. This implies that $V(F)=F$.

8.2. A remark on the Beauville-Debarre-Ran question. In $\S 6$ we saw that (2) implies (1) in Conjecture A. The full equivalence of (1) and (2) would have an amusing consequence for the equivalence of (1) and (5): it would imply that it is enough to check it only for subvarieties of dimension $d \leq\left[\frac{g}{2}\right]$ (or only for subvarieties of dimension $d \geq\left[\frac{g}{2}\right]$ ), i.e. essentially for half the dimensions involved. This follows from the proof of Theorem $\mathrm{B}$, where we saw that $\mathcal{I}_{X}(\Theta)$ is a $G V$-sheaf if and only if $\mathcal{I}_{V(X)}(\Theta)$ is a $G V$-sheaf, combined with the fact that on a Jacobian $J(C)$ we have $V\left(W_{d}(C)\right)=-W_{g-d-1}(C)$ up to translate (cf. Example 4.5). 
8.3. An addition to Conjecture $\mathbf{A}$ involving the theta-dual $V(X)$. Finally we make a comment, independent of the arguments above, emphasizing the fact that one can try to detect subvarieties of minimal cohomology class based directly on properties of $V(X)$. If we again identify $V(X)$ with the locus in $A$ parametrizing theta-translates containing $X$, then we have a difference map

$$
\phi: X \times V(X) \rightarrow X-V(X) \subset \Theta \subset A .
$$

Consider now the equalities

$$
\operatorname{deg}(\phi) \cdot[X-V(X)]=[X] *[V(X)]=\left(\begin{array}{c}
g-1 \\
d
\end{array}\right) \theta
$$

where $*$ denotes the Pontrjagin product on cohomology. These are satisfied for the difference maps $W_{d} \times W_{g-d-1} \rightarrow W_{d}-W_{g-d-1}$ and $F \times F \rightarrow F-F$, as for any $d$ we have the formula

$$
\frac{\theta^{g-d}}{(g-d) !} * \frac{\theta^{d+1}}{(d+1) !}=\left(\begin{array}{c}
g-1 \\
d
\end{array}\right) \theta
$$

(cf. [LB] Corollary 16.5.8). On the other hand, if (8) holds for some subscheme $X$ of dimension $d$, then $X$ has minimal class if and only if $V(X)$ has minimal class 6 This suggests adding a more geometric conjecture to the list in Conjecture $\mathrm{A}$ - namely under the hypotheses of Conjecture A, the following should be equivalent, and equivalent to the statements there:

(1) $\operatorname{dim} V(X)=g-d-1$.

(2) $V(X)$ has minimal cohomology class $[V(X)]=\frac{\theta^{d+1}}{(d+1) !}$.

(3) $[X] *[V(X)]=\left(\begin{array}{c}g-1 \\ d\end{array}\right) \theta$.

Note that (3) and (2) obviously imply (1). The key point is (1): subvarieties of minimal class should be characterized among nondegenerate subvarieties by the fact that their theta-dual variety has maximal dimension.

\section{REFERENCES}

[Be1] A. Beauville, Sous-variétés spéciales des variétés de Prym, Compositio Math. 45 (1982), 357-383.

[Be2] A. Beauville, Quelques remarques sur la transformation the Fourier dans l'anneau de Chow d'une variété abélienne, Proc. Jap.-Fr. Conf., Tokyo and Kyoto 1982, Springer LNM 1016 (1983), 238-260.

[CG] H. Clemens and P. Griffiths, The intermediate Jacobian of the cubic threefold, Ann. of Math. 95 (1972), $281-356$.

[De1] O. Debarre, Minimal cohomology classes and Jacobians, J. Alg. Geom. 4 (1995), 321-335.

[De2] O. Debarre, Fulton-Hansen and Barth-Lefschetz theorems for subvarieties of abelian varieties, J. Reine Angew. Math. 467 (1995), 187-197.

[EGA III] A. Grothendieck and J. Dieudonné, Eléments de Géométrie Algébrique, III, Étude cohomologique des faisceaux coherents, Publ. Math. IHES 11 (1961) and 17 (1963).

[EG] D. Eisenbud and S. Goto, Linear freee resolutions and minimal multiplicity, J. Algebra 88 (1984), 89-133.

[Fu] W. Fulton, Intersection theory, Second edition, Springer-Verlag, Berlin, 1998.

[GL] M. Green and R. Lazarsfeld, Deformation theory, generic vanishing theorems, and some conjectures of Enriques, Catanese and Beauville, Invent. Math. 90 (1987), 389-407.

[Hac] Ch. Hacon, A derived category approach to generic vanishing, J. Reine Angew. Math. 575 (2004), $173-187$.

[Hö] A. Höring, $M$-regularity of the Fano surface, preprint arXiv:0704.0558.

[Ho] William L. Hoyt, On products and algebraic families of jacobian varieties, Ann. of Math. 77 (1963), 415-423.

[LB] H. Lange and Ch. Birkenhake, Complex abelian varieties, 2nd edition, Springer-Verlag 2004.

\footnotetext{
${ }^{6}$ Here is a brief explanation: via the Fourier transform $F$ on cohomology, which is an isomorphism, the Pontrjagin product corresponds to the cup product. On the other hand, $F\left(\frac{\theta^{a}}{a !}\right)=\frac{\theta^{g-a}}{(g-a) !}$. But taking cup-product with this class is an isomorphism by the Lefschetz theorem. For details on all of this cf. e.g. [Be2], or [LB] $\S 16.3$.
} 
[Mu1] S. Mukai, Duality between $D(X)$ and $D(\widehat{X})$ with its application to Picard sheaves, Nagoya Math. J. 81 (1981), 153-175.

[Mu2] S. Mukai, Fourier functor and its application to the moduli of bundles on an abelian variety, In: Algebraic Geometry, Sendai 1985, Advanced studies in pure mathematics 10 (1987), 515-550.

[OP] W. Oxbury and C. Pauly, Heisenberg invariant quartics and $S U_{C}(2)$ for a curve of genus four, Mth. Proc. Camb. Phil. Soc. 125 (1999), 295-319.

[OSS] Ch. Okonek, M. Schneider, and H. Spindler, Vector bundles on complex projective spaces, Progr. Math., vol.3, Birkhäuser, 1980.

[PP1] G. Pareschi and M. Popa, Regularity on abelian varieties I, J. Amer. Math. Soc. 16 (2003), $285-302$.

[PP2] G. Pareschi and M. Popa, Castelnuovo theory and the geometric Schottky problem, to appear in J. Reine Angew. Math., preprint math.AG/0407370

[PP3] G. Pareschi and M. Popa, GV-sheaves, Fourier-Mukai transform, and Generic Vanishing, preprint math.AG/0608127.

[Ra] Z. Ran, On subvarieties of abelian varieties, Invent. Math. 62 (1981), 459-479.

[W] G. Welters, The surface $C-C$ on Jacobi varieties and 2nd order theta functions, Acta Math. 157 (1986), $1-22$.

Dipartamento di Matematica, Università di Roma, Tor Vergata, V.le della Ricerca ScienTIFICA, I-00133 Roma, ITALY

E-mail address: pareschi@mat.uniroma2.it

Department of Mathematics, University of Chicago, 5734 S. University Ave., Chicago, IL 60637, USA

E-mail address: mpopa@math.uchicago.edu 